\title{
Begleit- und Nachbehandlung von Beckenringverletzungen
}

\author{
Andreas Luther, Claudia Winkelmann, Karl Heinrich Winker
}

\section{Zusammenfassung}

Operationspflichtige Beckenringverletzungen treten gehäuft kombiniert mit Extremitäten- oder anderen Verletzungen auf. Thromboseprophylaxe, Infektprophylaxe und Physikalische Therapie stehen im Mittelpunkt der postoperativen Behandlung in der Klinikphase. Rehabilitation, klinische und radiologische Nachuntersuchungen, gegebenenfalls Folgeoperationen schließen sich an.

\section{Einleitung}

Die Begleit- und Nachbehandlung von Beckenringverletzungen beginnt mit dem Umlagern in der OP-Schleuse und endet in der sozialen und beruflichen Rehabilitation.

Abhängig vom Verletzungsmuster und dem Verlauf umfasst sie die initiale Betreuung auf der Intensivstation, die anschließende Klinikphase sowie die ambulante oder stationäre Rehabilitation. Gerade bei polytraumatisierten Patienten ist ein protrahierter Verlauf, gekennzeichnet durch Reeingriffe oder Korrekturperationen gelegentlich unvermeidbar. Nur die interdisziplinäre Teamleistung aller beteiligten Behandler und Betreuer unter Federführung des Unfallchirurgen garantiert ein optimales Behandlungsergebnis. [2,9]

\section{Operationstag}

Die initiale postoperative intensivmedizinische Überwachung nach einem Beckeneingriff ist angezeigt. Sie entbindet den Operateur nicht von der Verpflich-

OP-JOURNAL 2003; 19: 147-150

(c) Georg Thieme Verlag Stuttgart . New York tung seinen Patienten aufzusuchen, um folgende Informationen zu erlangen:

- Drainagemenge

- Durchblutungssituation, venöser Rückstrom (insbesondere nach ilioinguinalem Zugang)

- neurologischer Status

Die Operation endet nicht mit dem Wundverschluss oder dem Schreiben des Operationsberichtes, sondern mit der Nachmittagsvisite durch den Operateur am OP-Tag!

\section{Infektprophylaxe}

Die Infektprophylaxe beginnt mit der schonenden Operationstechnik.

Sie wird unterstützt durch die perioperative Antibiotikaapplikation. Die singleshot-Antibiose, bei Eingriffen über vier Stunden ergänzt durch eine zweite Gabe, ist ausreichend.

Sie ersetzt keinesfalls die sorgfältige Drainagepflege und die engmaschige klinische und sonographische Kontrolle des Wundgebietes.

Ein Antibiotikum ersetzt keinesfalls die Wund- und Drainagepflege!

Ultraschallkontrollen sollten am 4., 7. und 11. postoperativen Tag vorgenommen werden.

Bei einem sonographisch ermittelten Hämatomvolumen von mehr als $50 \mathrm{ml}$ besteht die Indikation zur Revision [12] (Abb. 1).

\section{Thromboseprophylaxe}

Beckeneingriffe gehören hinsichtlich der Thromboembolierate zu den Hochrisikoeingriffen.

Standard ist die gewichtsadaptierte Verabreichung eines niedermolekularen He-

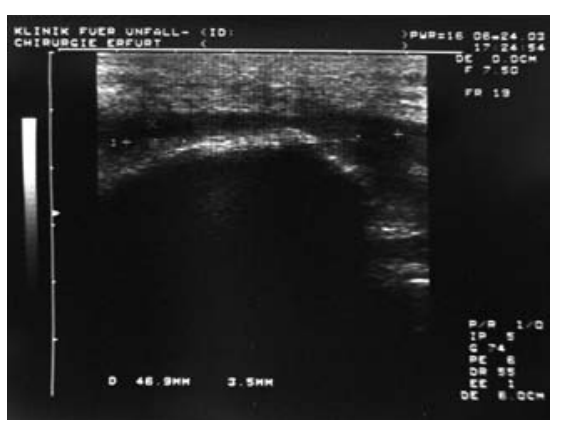

Abb.1 Sonographiebefund mit minimaler Hämatombildung.

parins. Die Dauer der Verabreichung richtet sich nach dem Mobilisationsgrad des Patienten und wird vom Gesamtverletzungsmuster bestimmt [9].

An dieser Stelle setzt die Physikalische Therapie ein. Durch statische und dynamische Muskelarbeit sollen kleine und mittelgroße Muskelgruppen im Sinne der lokalen aeroben Ausdauerverbesserung beansprucht werden.

Als passive Maßnahmen kommen venöse Streichmassagen und Bürstungen zur Anwendung $[6,11]$.

\section{Atemtherapie}

Bettlägerigkeit erhöht das Pneumonierisiko. Faktoren sind:

- Stase des Blutstroms

- Bewegungsmangel

- Hypoventilation

- reduzierte Atemexkursion.

Eine gezielte Therapie verhindert Sekretstau, Hypostase und Atelektasen.

Die medikamentöse Therapie spielt hierbei eine untergeordnete Rolle.

Physikalische Maßnahmen der Atemtherapie stehen im Vordergrund und bestimmen wesentlich die Prognose. Dazu zählen: 
1. Verbesserung der Atembewegungen zur Eröffnung von Atelektasen, Verlangsamung und Vertiefung der Atmung.

2. Lagerungswechsel zur Verbesserung der Lungendurchblutung, wobei die Schwerkraft zur Umverteilung des Lungenblutes genutzt wird.

3. Unterstützung des Sekrettransportes, Provokation des Hustens und Erleichterung des Abhustens. $\mathrm{Zu}$ den Maßnahmen gehören Thoraxvibrationen, Inhalationen und Totraumvergrößerung [6].

Gezielte Atemtherapie hilft eine Pneumonie zu verhindern und bestimmt somit wesentlich die Prognose!

\section{Dekubitusprophylaxe}

Durch Lagerungskontrolle, Lagerungswechsel und gezielte Übungen zur Anspannung der Gesäßmuskulatur lassen sich Dekubitalulzera vermeiden.

Die Hüftgelenke sollen möglichst in Nullstellung gelagert werden.

Dekubitusprophylaxe beginnt mit der Lagerung auf dem Operationstisch. Verantwortlich ist der Operateur!

\section{Erhalten der Armkraft}

Die Maßnahmen dienen der Vorbereitung für das Gehen an Unterarmstützen. Zur Anwendung kommen Formen des Bewegens und Haltens in komplexen Mustern als komplexe Widerstandsübungen.

\section{Schulung der Körperwahrnehmung}

In dieser Behandlungsphase erfolgt ebenfalls die Schulung der Körperwahrnehmung des Patienten, vor allem aufgrund der zentralen Stellung und funktionellen Anatomie des Beckens.

Hierzu gehören passive Griffe aus der Lösungstherapie nach Schaarschuch-Haase, myofasziale Releasetechniken und die Dekontraktion der Bauchmuskulatur.

\section{Gezielte Physikalische Therapie}

\section{Biomechanik des Beckens}

Die Schwerkraft wird beim aufrechten Gang über das Becken auf die Hüftköpfe übertragen. Der Schwerpunkt des Körpers liegt bedingt durch die Beckenkippung und die S-förmige Aufrichtung der Wirbelsäule vor der Wirbelsäule. Es resultiert eine Y-förmige Kraftübertragung über das Becken auf die Beine. Die Trageund Haltefähigkeit des Beckens wird gewährleistet durch das Zusammenspiel zwischen Muskulatur, Bandstrukturen und den knöchernen Komponenten. Beim aufrechten Gang wirken auch Rotationskräfte auf das Becken, da der Drehpunkt des Beckens dorsal der Mittelpunkte der Femurköpfe liegt. Bei Beckenverletzungen wird das räumliche Gefüge des Beckens verändert. Der physiologische Kraftfluss ist unterbrochen, die „intrapelvine Zuggurtung“ gestört [7] (Abb. 2).

\section{Erhaltung der Beinkraft}

Bei der Auswahl der Bewegungsmuster muss bedacht werden, dass sich bei den Formen der Widerstandsarbeit Verstärkungsmuster aufbauen. Diese können scherend wirken und beispielsweise vertikal verlaufende Frakturen irritieren [6] (Abb. 3).

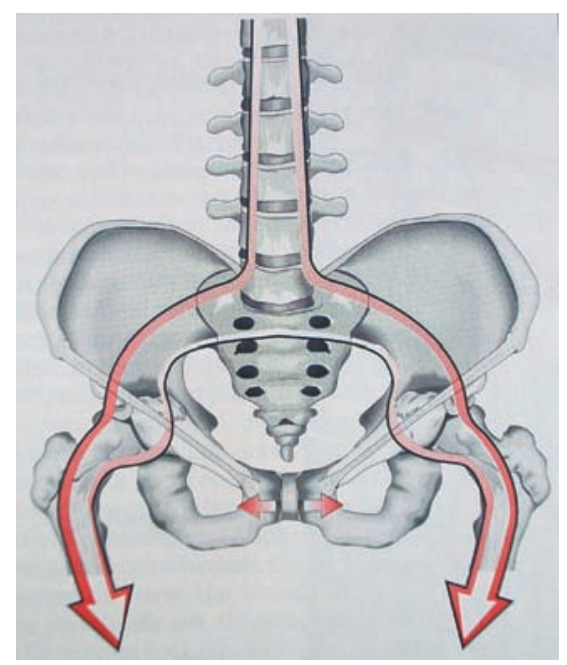

Abb. 2 Kraftfluss und Tragezonen am Becken [7].

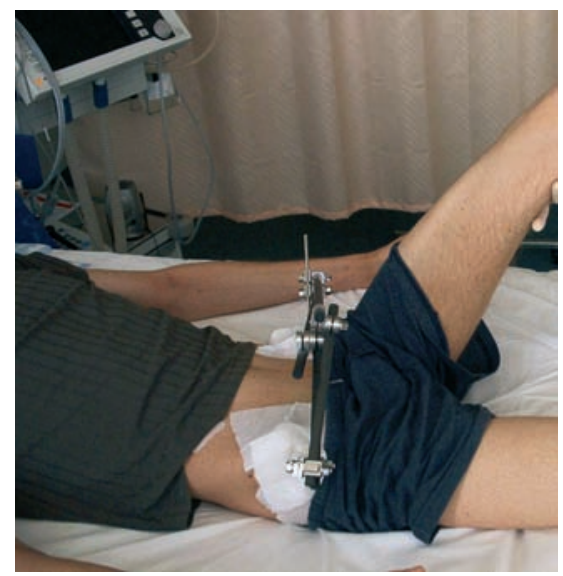

Abb. 3 Muskelkräftigung

\section{Aktive Stabilisation des Beckenringes}

Voraussetzung ist eine übungsstabile Osteosynthese bzw. eine primär stabile Fraktur.

Isometrische Spannungsübungen der ventralen und dorsalen Muskelketten zwischen Rumpf und Bein stabilisieren das Becken. Dazu werden in korrigierter Mittellage diagonale Spannungen aufgebaut. Das Herausschieben der Ferse gegen Führungskontakt stabilisiert die Beckenabduktion.

Hubarmen Bewegungen, die ihren Ursprung im Konzept der Funktionellen Bewegungslehre nach Klein-Vogelbach haben, wird eine größere Bedeutung beigemessen als den isometrischen Spannungsübungen.

Bei zunehmender Konsolidierung werden die PNF-Beckenmuster bei aktiver Fixation des Femurs ausgeführt $[6,11]$.

\section{Kräftigung der Glutäalmuskulatur}

Die atrophierte Glutäalmuskulatur wird durch Stabilisationsübungen, aber auch durch dynamische Übungen in konzentrisch oder exzentrisch dynamischer Spannungsform gekräftigt.

Aus der Bauchlage oder dem Vierfüßlerstand heraus kann geübt werden, wenn Bewegungsübergänge gestattet sind.

Erhalten der Gelenkbeweglichkeit in den Hüft-, Knie- und Sprunggelenken

Aktive Entspannungstechniken wirken der Kontrakturentstehung entgegen. Wichtig erscheinen die Spitzfußprophylaxe und Fußheberkräftigung.

Am Hüftgelenk werden schwerpunktmäßig die Extension und Innenrotation mobilisiert.

Kontraktur- und Spitzfußprophylaxe in der Frühphase auf der Intensivstation!

\section{Vorbereitung der Belastungsphase}

Hierzu dient zunächst das Üben des Transfers in die Seitenlage. Die vermehrte Instabilität, die in dieser Ausgangsstellung gegenüber der Rückenlage zu erwarten ist, provoziert Muskelaktivitäten. Insbesondere das Rekrutieren der autochthonen Muskulatur im Rumpfbereich ist für die späteren Ausgangsstellungen 


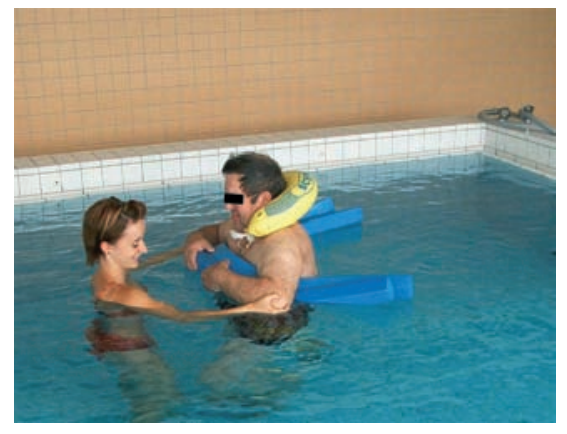

Abb.4a Aquajogging vor Erreichen der Belastungsstabilität, B2.2-Verletzung bei Adipositas per magna, Symphysenverplattung.

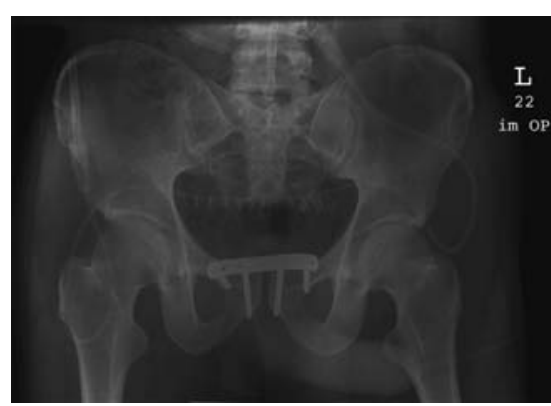

Abb.4b Postoperative Beckenübersichtsaufnahme.

Sitz, Stand bis hin zum Gang, wesentlich. Die Mobilisation im Wasserbad bietet eine weitere Möglichkeit, die Phase des Belastens und Gehens vorzubereiten, sofern die Wundverhältnisse keine Kontraindikation darstellen. Der Auftrieb gestattet das Trainieren des Gehmusters bereits vor dem Erreichen der Belastungsstabilität (Abb. 4a u. b).

\section{Belastungsphase}

Sie wird vorbereitet durch gezielte Vorübungen des Gehmusters. Der Zweioder Dreipunktgang wird geschult. Die Mehrzahl der Osteosynthesen am Becken gestattet früh eine Belastung mit $20 \mathrm{~kg}$ im Sinne des funktionellen Gehens. Eine Orientierung geben die

Entlastungszeiten nach Weise und Weller.

- Frakturen mit stabilen Osteosynthesen können in der 2.-4. postoperativen Woche schrittweise belastet werden.

- Für vertikale Beckenfrakturen mit stabilen Osteosynthesen werden 8-10 Tage Bettruhe empfohlen, anschließend Bewegungsbad und Teilbelastung je nach Schmerzsituation und Muskelfunktion.

- Bei Beckenverletzungen mit Azetabulumfrakturen und Osteosynthesen Bettruhe über 7-10 Tage, Teilbelastung für 6 Wochen.

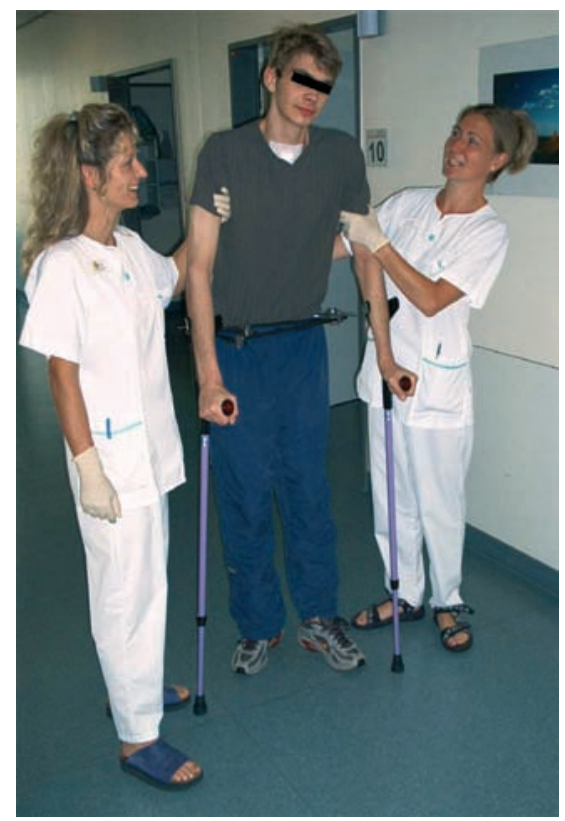

Abb. 5 Mobilisation unter Sicherung der B2Fraktur mit dem supraazetabulären Fixateur.

- Bei vorderer Beckenringfraktur ohne Stabilitätsverlust Aufstehen mit voller Belastung wenige Tage nach dem Unfall [6].

Meist ist zunächst der sichere Umgang mit Gehstützen oder Gehwagen erforderlich.

Bei der Belastungssteigerung ist auf Ermüdungszeichen, Schmerzen und Ausweichbewegungen wie Duchenne- oder Trendelenburgzeichen zu achten [6].

Bei B-Frakturen ist mit angelegtem supraazetabulären Fixateur eine forcierte Belastungssteigerung möglich (Abb.5).

Einen erheblichen Teil des Therapieprogramms nimmt die Erarbeitung der Standbeinphase ein. Sie reicht bis in die Rehabilitationsphase und umfasst PNFTechniken und die Nutzung beweglicher Unterlagen, speziell das Training auf dem Therapiekreisel und der Weichbodenmatte.

Belastungssteigerung nicht nur vom radiologischen Befund, auch von Ermüdungszeichen, Schmerzen, Ausweichbewegungen abhängig machen!

\section{Sportmedizinische Aspekte}

Mit Ausnahme von Rasanztraumen sind knöcherne Beckenverletzungen als Folge von Sportunfällen selten.

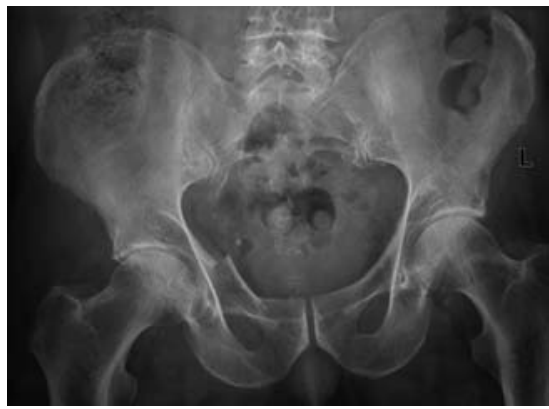

Abb. 6 A2-Fraktur eines 82-jährigen Patienten.

Meist sind Muskeln, Bänder und Sehnen betroffen.

Bei den Frakturen dominieren hier die Abrissfrakturen (Typ A), häufig in Form von Apophysenabrissen. Da sie im Gegensatz zu den Epiphysen keinen Einfluss auf das Längenwachstum haben, sind sie eine Domäne der konservativen Therapie.

Sie besteht in der

- Teilbelastung bis zur Schmerzfreiheit

- Kryotherapie

— individuellen Übungsbehandlung [4]

\section{Beckenfrakturen im Alter}

Die Altersverteilung bei Beckenfrakturen zeigt drei Altersgipfel. Charakteristisch für den dritten Gipfel zwischen dem 70 . und 85.Lebensjahr ist ein Überwiegen des weiblichen Geschlechts.

Die zumeist harmlosen transpubischen Altersfrakturen werden regelmäßig durch die hohe Komorbidität kompliziert.

Die sehr seltenen instabilen Beckenverletzungen im Alter stellen eine akute Lebensgefahr dar, weil allein der Blutverlust aufgrund der fehlenden Kompensationsmöglichkeiten im Alter limitierend wirken kann [2] (Abb. 6).

Die Therapie beruht auf vier Säulen:

- Schaffung der Voraussetzungen für die gezielte Mobilisation

- Wiedererlangung der Fähigkeiten im Sinne der Aktivitäten des täglichen Lebens

- Osteoporosetherapie (Kalzium, Vitamin D und je nach Genese der Osteporose ggf. Bisphosphat oder Östrogenrezeptorblockade)

- Analyse und Prohylaxe der Sturzursache

Im eigenen Patientengut verfügen wir über sehr gute Erfahrungen in der früh- 
zeitigen gemeinsamen Betreuung der Patienten durch den Geriater und den Unfallchirurgen.

Eine frühzeitige interdisziplinäre Zusammenarbeit zwischen dem Unfallchirurgen und dem Geriater ermöglicht die gezielte Mobilisation, die Wiedererlangung der ATL, die Analyse der Sturzursache und die Prophylaxe weiterer Frakturen!

\section{Rehabilitation}

Der Unfallchirurg in der Klinik hat eine besondere Verantwortung für die Einleitung einer erforderlichen Rehabilitation, insbesondere für eine rechtzeitige Aufklärung und Beratung $(1,20,40,73$ SGB V, Beratungs-, Mitwirkungs- und Meldepflichten des Arztes).

Ziel und Zweck der Rehabilitation ist es, den Behinderten oder von Behinderung Bedrohten möglichst auf Dauer in Arbeit, Beruf und Gesellschaft einzugliedern.

Nach Beckenfrakturen sind von Bedeutung:

1. Medizinische Maßnahmen

ärztliche Behandlung

a physikalische Therapie (ambulant/ stationär)

Ausstattung mit orthopädischen Hilfsmitteln

2. Berufsfördernde Leistungen

- Belastungserprobung

berufliche Anpassung und Umschulung

- Förderung der Arbeitsaufnahme

3. Leistungen zur sozialen Rehabilitation und ergänzende Leistungen

- Haushalthilfe

- Kraftfahrzeughilfe [8]

\section{Nachuntersuchung nach Beckenfrakturen}

Während noch in der jüngeren Vergangenheit das Überleben einer schweren Beckenverletzung als wesentlicher Behandlungserfolg angesehen worden ist, steht zunehmend die komplette Restitutio auch nach komplexen Verletzungen in der Diskussion.

Um die Prognose von Beckenverletzungen, auch unter dem Aspekt neurologischer und urologischer Störungen, verifi- zieren zu können, sollten die Patienten auf langfristige Nachuntersuchungstermine nach Abschluss der Primärbehandlung hingewiesen werden [5].

Es gilt anhand gängiger Klassifikationen (AO-Klassifikation, Hannoveraner Verletzungsschlüssel) für den individuellen Behandlungsfall zukünftig eine Prognose hinsichtlich des Langzeitergebnisses angeben zu können [10].

Nach Gänsslen und Pohlemann sollte die Nachuntersuchung folgende Punkte beinhalten [5]:

\section{Nachuntersuchung nach \\ Beckenverletzung}

Anamnese

(Schmerzen, Visual-Analog-Skala)

körperliche Untersuchung

(Gangbild, Beinlänge,

Hüftbeweglichkeit, Fehlstellungen)

- orientierende neurologische

Untersuchung

(Motorik, Sensibilität)

- orientierende urologische

Untersuchung

(Miktionsstörungen, Erektions-

störungen etc.)

- radiologische Untersuchung

(Beckenübersichtsaufnahme,

Inlet- und Outletprojektion, CT)

\section{Begutachtung nach Beckenverletzungen}

Beckenverletzungen treten gehäuft bei Mehrfach- und polytraumatisierten Patienten auf. Es besteht eine Korrelation zwischen der Häufigkeit von Spätfolgeschäden und der primären Instabilität der Verletzung [5].

Verletzungsfolgen am Becken sind besonders schwer zu objektivieren. Klinische, radiologische und computertomographische Untersuchungen haben einen hohen Stellenwert. Einteilungskriterium ist ana$\log$ zur AO-Klassifikation die Integrität des dorsalen sakroiliakalen Ringsegmentes. Sein Stabilitätsverlust korreliert mit der Funktionseinschränkung. Ausdruck der Instabilität ist die symphysale Diastase. Folglich findet diese gutachterlich bei der Einschätzung der MdE Anwendung $[3,5]$.

Neurologische und urologische Zusatzgutachten sind oft unabdingbar.

\section{Literatur}

${ }^{1}$ Beckers D, Deckers J. Ganganalyse und Gangschulung: Therapeutische Strategien für die Praxis, Rehabilitation und Prävention, Bd. 38, Springer 1997

2 Culemann U, Reilmann H. Verletzungen des Beckenringes. Der Unfallchirurg, o.J., H. 6, S 487-496

${ }^{3}$ Echtermeyer V, Sangmeister M, Lange K. Begutachtung nach Beckenverletzungen aus chirurgischer Sicht In Gutachten Kolloquium 10. Springer, Berlin-Heidelberg-New York, 1995

${ }^{4}$ Gänsslen A, Hüfner T, Reilmann H, Pohlemann T, Weinberg A, Wippelmann B. Besondere Situationen am Beckenring, In Tscherne $\mathrm{H}$, Pohlemann T, Siehe 3

${ }^{5}$ Gänsslen A, Müller-Vahl $\mathrm{H}$, Pohlemann T, Stief CH. Langzeitergebnis nach Beckenfrakturen. In: Tscherne H, Pohlemann T, Unfallchirurgie, Becken und Azetabulum. Springer, Berlin Heidelberg New York 1998

${ }^{6}$ List M. Physiotherapeutische Behandlung in der Traumatologie. Springer Berlin Heidelberg 1999

${ }^{7}$ Mutschler W, Haas N. Praxis der Unfallchirurgie. Thieme, Stuttgart New York 1999

${ }^{8}$ Rompe G, Erlenkämper A. Begutachtung der Haltungs und Bewegungsorgane. Thieme, Stuttgart New York 1998

9 Tscherne H, Regel G. Unfallchirurgie, Traumamanagement. Springer, Berlin Heidelberg 1997

${ }^{10}$ Weber K, Müller W, Wentzensen A. Lebensqualität nach operativ behandelten Beckenringfrakturen. Unfallchirurg 2001; 104: 1162-11677 Springer 2001

${ }^{11}$ Weimann G. Physikalische Therapie. Hippokrates Stuttgart 1993

${ }^{12}$ Ziegert A, Petereit U, Winker KH. Sonographisch assistierte Verlaufskontrolle operativ versorgter koxaler Femurfrakturen, Aktuelle Traumatologie, 28.Jahrgang, Heft 2 April 1998, Thieme Stuttgart

\section{Dr. med. Andreas Luther \\ Oberarzt}

Claudia Winkelmann

Leiterin der Abteilung Physikalische Medizin und Rehabilitation

Prof. Dr. med. Karl Heinrich Winker Chefarzt

Klinik für Unfall-, Hand- und Wiederherstellungschirurgie HELIOS Klinikum Erfurt

Nordhäuser Str. 74

D-99089 Erfurt 